\title{
THE SEARCH OF SPATIAL FUNCTIONS OF PRESSURE IN ADJUSTABLE HYDROSTATICRADIAL BEARING
}

\author{
Dmytro FEDORYNENKO*, Sergiy BOYKO*, Serhii SAPON* \\ *Mechanical Engineering Department, Chernihiv National University of Technology, \\ 95 Shevchenka Str., 14027 Chernihiv, Ukraine \\ fdy@mail.ru, svboyko.cstu@gmail.com, s.sapon@gmail.com
}

\begin{abstract}
The analysis of spatial functions of pressure considering the geometrical deviations and the elastic deformation of conjugate surace have been considered. The analysis of spatial functions of pressure is performed by the finite element method. The difference of the size of pressure in a tangential direction of a pocket of a support under various service conditions has been investigated. A recommendation for improving of operational characteristics in regulated hydrostatic radial bearing has been developed.
\end{abstract}

Key words: Hydrostatic Bearing, Lubricating Film, Spatial Functions, Hydromechanics, Reynold's Equation

\section{INTRODUCTION}

The main characteristics to calculate the hydrostatic bearing $(\mathrm{HB})$, as well as other types of sliding bearings is a function of the pressure distribution. From its solution depends on the accuracy of calculations. Therefore, the calculation of the pressure distribution in the lubricating film HB has been given much attention.

The basis of calculation of the pressure distribution in the lubricating film is the basic equation of Hydromechanics (Michael, 2012):

- Navier-Stoke's

$$
\frac{\partial \vec{V}}{\partial t}+(\vec{V} \nabla) \vec{V}=F-\frac{1}{\rho} \operatorname{grad}(p)+v \nabla^{2} \vec{V}
$$

_ indissolubility of flow

$$
\operatorname{div} \rho \vec{V}=0
$$

where: $\vec{V}$ - particle fluid velocity vector in the point of space with the coordinates $x, y, z$ at a certain time $t ; \nabla$ - Hamiltonian operator; $F$ - volume forces acting on a fluid particle; $\rho, v$ - density and coefficient of kinematic viscosity of the fluid; $\nabla^{2}-$ Laplace operator.

For isothermal ( $v=$ const. ) laminar, steady motion of a viscous and uncompressed fluid $(\operatorname{div} \rho \vec{V}=0, \rho=$ const) considering order of magnitude in the previous equations, we can write in the Cartesian coordinate system:

$\frac{\partial p}{\partial x}=\mu \frac{\partial^{2} u}{\partial y^{2}} ; \frac{\partial p}{\partial y}=0 ; \frac{\partial p}{\partial z}=\mu \frac{\partial^{2} w}{\partial z^{2}} ;$

$\frac{\partial u}{\partial x}+\frac{\partial v}{\partial y}+\frac{\partial w}{\partial z}=0$

where: $\mu$ - coefficient of the fluid dynamic viscosity; $u, v, w-$ projection of fluid particle velocity vector on the axes $o x, o y, o z$ respectively.

By integrating the continuity equation (the last equation in system (3)) to $\boldsymbol{y}$ within the limits the clearance in the HB considering boundary conditions (Kiogora et al., 2014) we get the equation for the Reynold's pressure in the radial HB.
Analyzing the research conducted in Kiogora et al. (2014), it can be concluded that the final Reynold's equation in a cylindrical coordinate system is a linear non homogeneous differential equation in partial derivatives of second order elliptic type with two independent variables $\varphi, \bar{z}$ (angle of working chamber in HB and working length, respectively). In general case this equation has no analytical solution. Therefore, for the approximate decision of Hydromechanics tasks and study of the static characteristics of HB in practice various methods are used: finite differences, variation methods for differential equations in partial derivatives, finite element method and finite volumes (McDonald, 1971). The most common - finite volume method, which is described in Strutynsky and Fedorynenko (2011).

\section{ANALYSIS OF RECENT RESEARCHES AND PUBLICATIONS}

The most convenient for the numerical solution of Reynold's equations is the method of finite differences, with which you can get a set of discrete values of the approximate solution of differential equations in the investigated area, which is covered by mesh. According to this method the differential task is replaced by finite differences and difference solution is defined on the mesh.

In Strutynski and Fedorynenko (2011) this method is used for the numerical solution of Reynold's equation and static characteristics of $\mathrm{HB}$. In this paper the reamer of the working chamber bearing on the plane has been investigated. Using the method of finite differences differential Reynold's equation has been approximated and the second order partial derivatives have been replaced by their difference analogous. To solve received systems of equations were used iterative methods which compared with direct, have a number of advantages, first of all, these methods require fewer arithmetic operations to achieve a solution and quite easily are programmed on a computer.

The result of the calculation is the spatial pressure diagram $\bar{p}(\varphi, \bar{z})$ on Fig. 1 built for the domain of the function, which is limited by the working chamber. $\varphi-$ Is the angle of the bearing working chamber, $\bar{z}-$ the geometric dimensions of the working chamber. 
The method of finite differences considered in Strutynski and Fedorynenko (2011) for the numerical solution of Reynold's equation and static characteristics of HB requires the development of complex mathematical models and complex calculations.

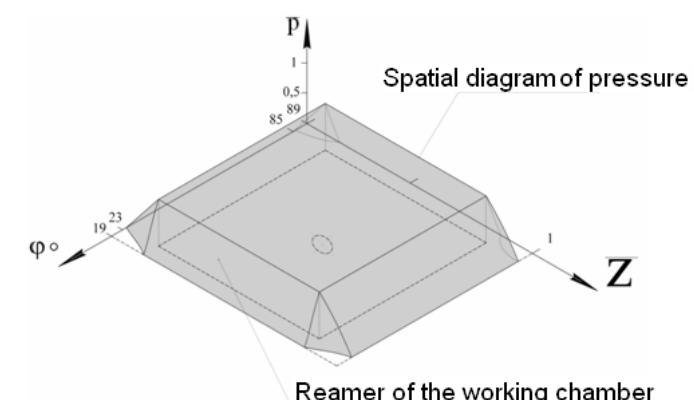

Fig. 1. Spatial diagram of the pressure which is obtained by the method of finite differences

The purpose of the paper is the analysis of spatial functions of pressure considering the geometrical deviations and the elastic deformation of conjugate surface by the finite element method and development of recommendations for improving of operational characteristics in regulated hydrostatic radial bearing.

\section{METHODS}

The most common approaches to the numerical solution of differential Reynold's equations, besides, considered method of finite differences - is the finite elements method and finite volumes. These methods are based on discretization liquid medium finite element, which is the solution of the problem by the set of interpolation functions. Finite volume method is a particular, quick case of the finite element method (McDonald, 1971).

To solve this problem effectively has been proposed the usage of modern CFD software packages in particular CosmosFloWorks. In this software the fluid motion is modeled by using Navier-Stoke's equations which describe the laws of momentum conservation, mass, energy, fluid environment. To search a solution of this problem is used discretization of Navier-Stoke's equations on the surface of finite volume of a computational grid. More detailed foundations of the method of finite volumes and algorithms for finding solutions on discrete meshes have been covered in the monographs Reddy and Gartling, (2010).

Let us consider the approaches to finding spatial functions of pressure in adjustable radial HB by means of CFDCosmosFloWorks.

The object of analysis is an adjustable radial $\mathrm{HB}$, basic design of which is shown in Fig. 2.

The design of the adjustable radial HB consists of the following elements: 1 - case, 2 - conical bushing, 3 - hydrostatic bushing, 4 - working chamber, 5 - cover, 6 - nut, 7 - choke, 8 - spindle, 9,10 - bulkheads.

Assumptions as for the model of hydrodynamic analysis are as following (Fedorynenko, 2012):

- The movement of fluid in the flow area of HB can be attributed to "slow" flow of liquid. Therefore, the forces of inertia and mass forces are negligible compared with the pressure forces and forces caused by the viscosity of the liquid.

- For medium and high speed HB with $V_{\varphi}<70 \mathrm{~m} / \mathrm{s}\left(V_{\varphi}\right.$ - linear speed of a spindle bearing surface in the tangential direction) and $\delta_{0}>10$ microns $\left(\delta_{0}-\right.$ backlash) it is possible to use the problem in the isothermal formulation with the averaged values of the temperature in the flow holes of the bearing.

Lubricant - Newtonian fluid, the pressure of lubricant layer is constant, sliding on the boundaries of a backlash and the gap in the liquid layer are absent (accepted Sommerfeld hypothesis), the thickness of the lubricant layer is small compared with the radius of the bearing, lubricant viscosity is accepted with some averaged temperature, the gradient of pressure in working chamber is zero.

a)

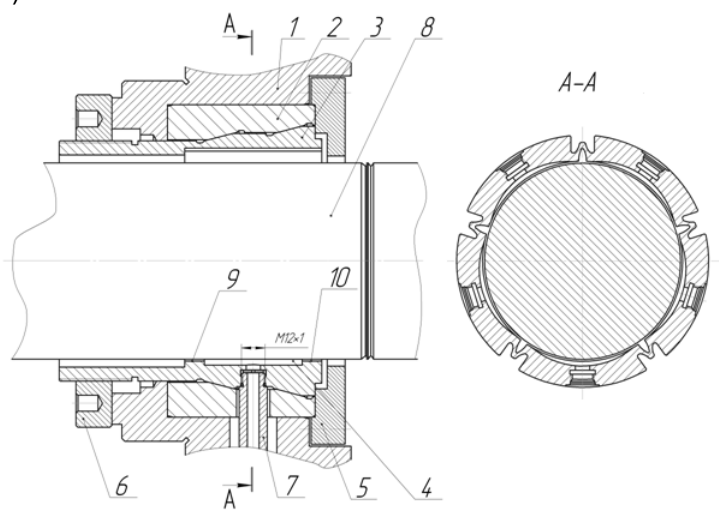

b)

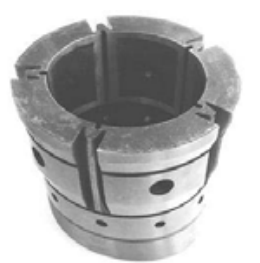

Fig. 2. Drawing of adjustable radial $H B$ a) and appearance $b$ )

For calculations were used solid-state 3D model of the hydrostatic bearing. To solve the problem were taken into account geometric deviation of bearing surfaces in the tangential direction of HB by identifying the cross section of shapes of the spindle bearing surface, which corresponds to the function of the radial backlash with deviations geometry of conjugate surface (Strutynski and Fedorynenko, 2011).

To determine the calculated domain (the domain of function definition) it was necessary to specify its boundary in space. The boundary of the calculation is the bearing surface of HB. In order to simulate the fluid flow through the holes in the solid model were used special covers (Sahno et al., 2009), which are shown in Fig. 3. The surface of these covers is interpreted in CosmosFloWorks as a hole on the inner surface (on Fig. 3 are shown by arrows) that define the boundary conditions.

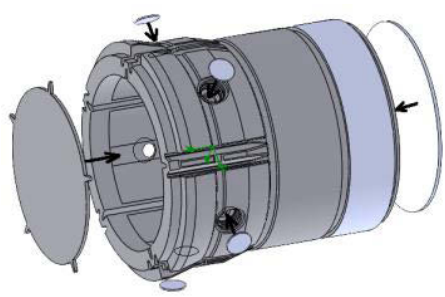

Fig. 3. Entering of boundary conditions on the 3D model (the spindle is not shown) 
On the input covers (corresponding to apertures for supplying the liquid in the chamber of $\mathrm{HB}$ ) was specified the static value of fluid pressure $p_{k_{i}}$. The output covers are analogues of holes on the end surfaces of the bearing, on the inner surfaces of which was set the drain pressure $p_{d}$ The boundary condition on the supporting surface of the spindle is the rotational speed, which is $n_{s}=2000 \mathrm{~min}^{-1}$.

As a result of the calculation was obtained the spatial diagram of the pressure on the supporting surface of the $\mathrm{HB}$, which is presented in Fig. 4.

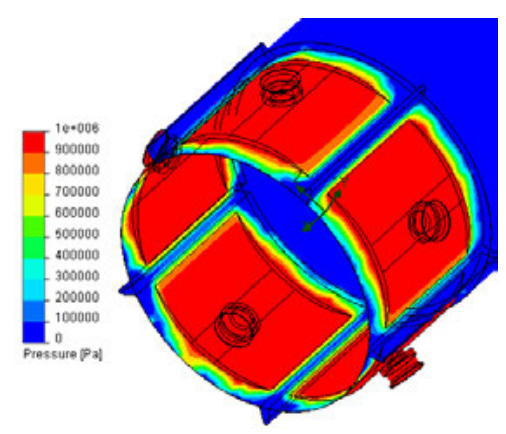

Fig. 4. The spatial diagram of the pressure on the supporting surface of adjustable radial $\mathrm{HB}$

The format of the data in Fig. 4 provides only a qualitative picture of the pressure distribution in the bearing. Quantitative evaluation of the scalar field of the pressure at some points of liquid medium using spatial diagrams is more complicated. To determine the fluid pressure in the specific sections of HB was proposed the age of spatial grid of sketches (Fig. 5), covering the study area of the bearing surface in the axial and tangential directions.

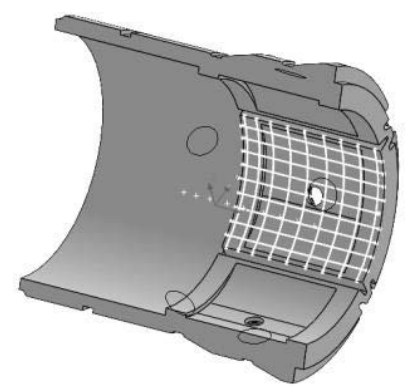

Fig. 5. The grid of sketches for analysis functions $p(\phi, z)$ on the area limited by a separate chamber of bearing

By the means of CosmosFloWorks for each sketch was determined fluid pressure dependence from the length of the line sketch. In Fig. 6 are shown dependences of fluid pressure, as defined by the length of the sketches in the axial (Fig. 6a) and tangential (Fig. 6b) directions of $\mathrm{HB}$.

By the means of a grid of sketches (see Fig. 5) were constructed the spatial diagrams of pressure, which is shown in Fig. 7.

Let us compare the results of calculations of differential $\mathrm{Na}$ vier-Stoke's equations by the method of finite differences and finite volumes.

The function of the pressure $p(\varphi, z)$ along the length of the tangential bulkheads in the axial direction of the bearing (see Fig. 1 and Fig. 6, 7) is qualitatively and quantitatively similar for both methods of numerical calculation.
There are also differences in the obtained solutions. On the end of the bearing surfaces of $\mathrm{HB}$ and the edges of drainage grooves in modeling functions of pressure by means of CosmosFloWorks were received non-zero values of fluid pressure (Fig. 6)as opposed to problem solution by finite differences, where the fluid pressure was equal to zero. Along the length of chambers in the tangential direction of the bearing the pressure difference is observed (see Fig. 6 and 7), which is caused by the rotation of the spindle, which does not meet the boundary conditions with the constant pressure value $p_{k_{i}}$ if finding problem solution by finite differences (see Fig. 1). It was proved that the increase of fluid pressure in tangential length of the bearing chambers takes place in the direction of rotation of the spindle (Sakhno et al., 2009; Fedorynenko et al., 2013).

a)

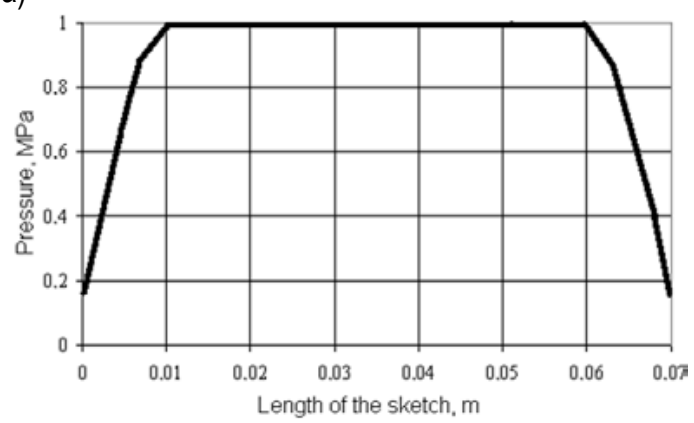

b)

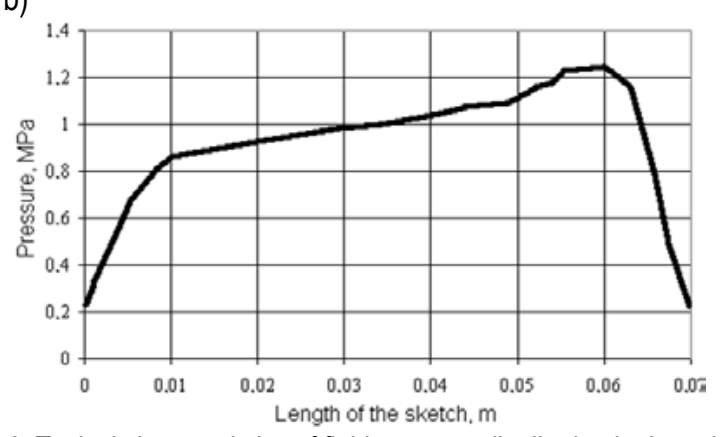

Fig. 6. Typical characteristics of fluid pressure distribution in the axial a) and tangential b) directions of $\mathrm{HB}$

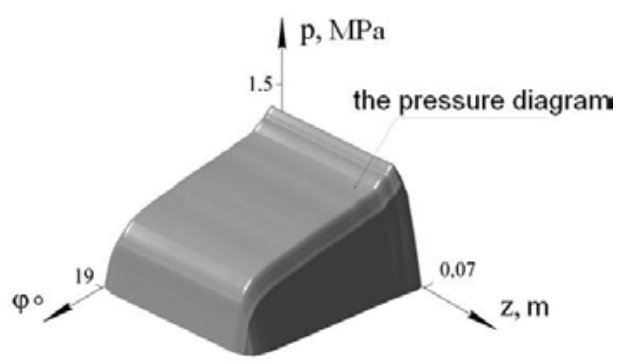

Fig. 7. The function of fluid pressure $p(\phi, z)$ in the chamber of $\mathrm{HB}$

The pressure difference in the $i-$ th chamber caused by the rotation of the spindle can be characterized by a dimensionless quantity $\Delta \bar{p}_{i}$ that is defined as:

$\Delta \bar{p}_{i}=\frac{\left|p\left(\varphi_{\tau k_{1}}, z_{c}\right)-p\left(\varphi_{\tau k_{2}}, z_{c}\right)\right|}{p_{k_{i}}}$

As a result of calculation by formula (4) were built the graphs of the pressure difference in the chamber of adjustable $\mathrm{HB}$, which are shown in Fig. 9. 
As shown in Fig. 9, increasing of fluid pressure supplied to the working chamber, leads to a decrease of pressure difference during spindle rotation in the bearing. It was found that with increasing frequency of spindle rotation $n_{s}$ the pressure difference in the chamber of the bearing increases and at lower values $p_{k_{i}}$ those facts are more intense.

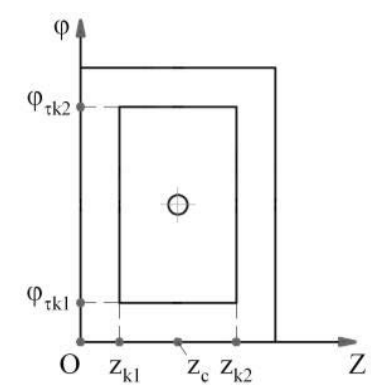

Fig. 8. Definition of a pressure difference in the $i$-th chamber

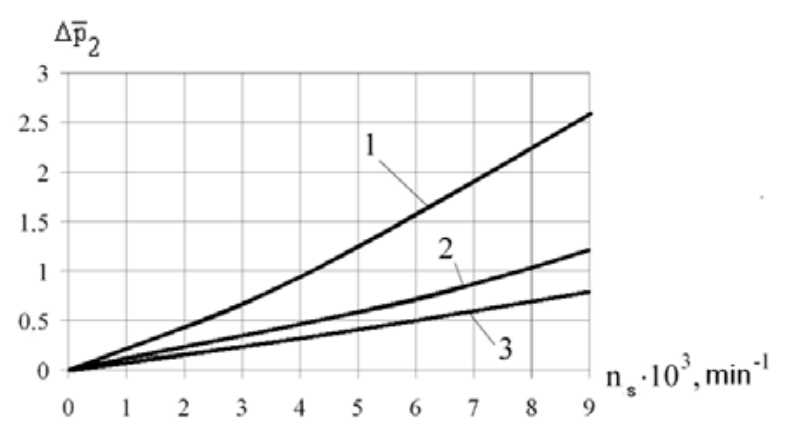

Fig. 9. Pressure difference on tangential length of the second working chamber: $1-p_{k_{2}}=1 \mathrm{MPa} ; 2-p_{k_{2}}=2 \mathrm{MPa}$; $3-p_{k_{2}}=3 \mathrm{MPa}$

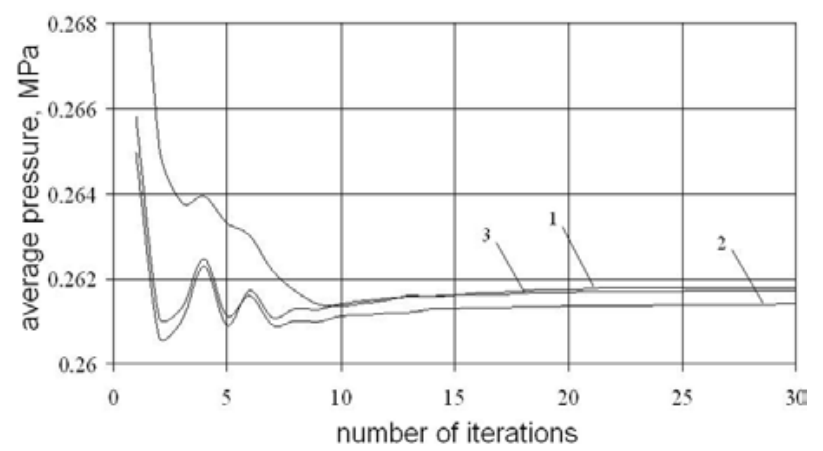

Fig. 10. Assessment of the accuracy of the calculation of the pressure function by means of CFD CosmosFloWorks:

$1-N_{K}=2383 ; 2-N_{K}=13192 ; 3-N_{K}=20443$

The estimation of the accuracy of the calculation of the pressure function by the finite volumes can be performed by comparing the calculation results with different grids, which differ in a size and a number of cells.Solution of stationary problems inCosmos FloWorks are based on the iterative algorithms with averaging of the results of calculation time. The criterion for completion of the iterative process is the convergence of the calculation to some stable decision in time. For determination of this point, certain criteria are used: physical parameters of the system that can characterize the condition of the calculation in time. If during calculating the value of the criterion has behavior close to the stationary, a decision on the completion of the search process iterative solution of the problem is made (Fedorynenko, 2009).
In this paper, a criterion for the completion of the iterative process of the calculation is the value of the average pressure in a predetermined liquid volume. The graphs that characterize mesh convergence calculation in different sizes and quantities $N_{K}$ cells are shown in Fig. 10.

The graph shows that the value of the average pressure in the calculation field matches some constant value of pressure after 20 iterations. The maximum relative difference of calculating data values of average pressure when using grids with different numbers of cells does not exceed $0.11 \%$, indicating a satisfactory accuracy of the calculation.

\section{RESULTS}

As a result of simulation of output characteristics of the controlled radial HB, it has been discovered that the greatest influence on these characteristics was caused by geometric deviation bearing surfaces in the tangential direction of the bearing and bias of the spindle axis in HB.

In order to improve the bearing capacity of HB during machining processing it is necessary to raise the pressure in the chamber $p_{k}$ and reduce the radial backlash in the bearing $\delta_{0}$.

Changing the pressure in the chamber of $\mathrm{HB}$ is limited by the preservation of optimal pressure differential $\Delta p\left(\Delta p=p_{H}-p_{k}\right)$ for maximum rigidity layer lubrication and maximum pressure of pumping unit.

So the most convenient way to increase the bearing capacity is to regulate the size of the static radial backlash $\delta_{0}$, which can be used for variety of supply systems of supply in the modes as a constant flow rate $(Q=$ const), and constant fluid pressure $(p=$ const $)$.

\section{REFERENCES}

1. Alyamovskyy A. (2005), SolidWorks. Computer modeling in engineering practice, BHV-Petersburg, St. Petersburg.

2. Fedorynenko D. (2009), Modeling Working Processes in the Controlled Hydrostatic Bearings with Means of CAD/CAE Systems, Journal of Chernihiv State Technological University, Series "Technical science", 37, 91 - 98.

3. Fedorynenko D. (2012), The spatial function of pressure in adjustable hydrostatic bearing, Journal of ChernihivStateTechnological University, Chernihiv, 2 (57), 63- 70.

4. Fedorynenko D., Sapon S., Boyko S. (2013), Precision Spindles with Adjustable Hydrostatic Bearings, Journal of National Technical University of Ukraine "Kyiv Polytechnic Institute", 3(69), 155-149.

5. Kiogora P. R., Kinyanjui M. N., Theuri D. M. (2014), A Conservative Scheme Model of an Inclined Pad Thrust Bearing, International Journal of Engineering Science and Innovative Technology, 3(01), 32-41.

6. McDonald P. W. (1971), The computation of transonic flow through two-dimensional gas turbine cascades, ASME, 71-GT-89, 12- 21.

7. Michael F. (2012), Towards a Navier Stokes Exact Solution,http://dx.doi.org/10.2139/ssrn.2193095.

8. Reddy J. N., Gartling D. K. (2010), The Finite Element Method in Heat Transfer and Fluid Dynamics, CRC Press.

9. Sakhno Y., Fedorynenko D., Boyko S., Volyk V. (2009), Adjustable Hydrostatic Bearings for Spindles, Publishing house "Aspect-polygraph", Nizhyn.

10. Strutynsky V., Fedorynenko D. (2011), Statistical dynamics of spindle units for hydrostatic bearings, LLC "Publishing" AspectPolygraph", Nizhin. 